\title{
A motivic study of generalized Burniat surfaces
}

\section{Chris Peters ${ }^{1}$}

Received: 26 March 2018 / Published online: 1 November 2018

(c) The Author(s) 2018

\begin{abstract}
Generalized Burniat surfaces are surfaces of general type with $p_{g}=q$ and Euler number $e=6$ obtained by a variant of Inoue's construction method for the classical Burniat surfaces. I prove a variant of the Bloch conjecture for these surfaces. The method applies also to the so-called Sicilian surfaces introduced by Bauer et al. in (J Math Sci Univ Tokyo 22(2-15):55111, 2015. arXiv:1409.1285v2). This implies that the Chow motives of all of these surfaces are finite-dimensional in the sense of Kimura.
\end{abstract}

Keywords Algebraic cycles · Chow groups · Finite-dimensional motives · Burniat surfaces $\cdot$ Inoue surfaces $\cdot$ Sicilian surfaces

Mathematics Subject Classification $14 \mathrm{C} 15 \cdot 14 \mathrm{C} 25 \cdot 14 \mathrm{C} 30 \cdot 14 \mathrm{~J} 29$

\section{Introduction}

Quite recently Bauer et al. [1] have investigated a generalization of Inoue's construction [7] of the classical Burniat surfaces [4]. These surfaces are minimal, of general type and have invariants $p_{g}=q=0,1,2$ or 3 .

Recall that the Chow group $\mathrm{CH}_{k}$ is said to be "trivial", if the natural cycle class map $\mathrm{CH}_{k} \hookrightarrow \mathrm{H}_{2 k}$ is injective. The kernel of the cycle class map $\mathrm{CH}_{k}^{\text {hom }}$ can be investigated by means of the Abel-Jacobi map $\mathrm{CH}_{k}^{\text {hom }} \rightarrow J^{k}$, where the target is the $k$ th intermediate Jacobian. Its kernel is denoted $\mathrm{CH}_{k}^{\mathrm{AJ}}$. If this vanishes, this has strong consequences. For instance for surfaces this implies $p_{g}=0$ and the Albanese map is an isomorphism up to torsion. The converse is Bloch's conjecture [3]. In a follow-up study [2] this conjecture has been verified for the generalized Burniat surfaces, i.e $\mathrm{CH}_{0}^{\text {hom }}=0$.

These generalized Burniat surfaces $Y=X / G$ are all quotients of $X$ by a freely acting abelian group $G \simeq(\mathbf{Z} / 2 \mathbf{Z})^{3}$ and where $(X, G)$ is a so called Burniat hypersurface pair $(X, G): X$ is a hypersurface in a product $A$ of three elliptic curves having at most nodes

Communicated by Daniel Greb.

Chris Peters

c.a.m.peters@tue.nl

1 Discrete Mathematics, Technische Universiteit Eindhoven, Postbus 513, 5600 MB Eindhoven, Netherlands 
as singularities and $G$ is an abelian group acting freely on $A$ and leaving $X$ invariant. The surface $X$ is also called Burniat hypersurface. These come in 16 families, enumerated $\delta_{1}-\delta_{16}$. The classical Burniat surface belongs to the 4-parameter family $\delta_{2}$. Also the family $\delta_{1}$ is 4 dimensional. The remaining families have only 3 parameters coming from varying the elliptic curve. This implies that the equation of $X$ in these cases is uniquely determined, contrary to the first two where there is a pencil of hypersurfaces invariant under $G$. The surfaces $Y$ have at most nodal singularities. For simplicity I assume in this note that $Y$, and hence $X$ is smooth, which is generically the case. However, none of the arguments is influenced by the presence of nodal singularities.

In [9] it has been remarked that the main theorem of loc. cit. yields the Bloch conjecture for the classical Burniat surfaces. The goal of this paper is to apply the same methods to all Burniat hypersurfaces. In particular, one obtains a short proof of the Bloch conjecture in the appropriate cases.

To state the result, let me recall that the Chow motive ${ }^{1} \mathrm{~h}(X)$ is the pair $(X, \Delta)$ where $\Delta \subset X \times X$ is the diagonal considered as a (degree 0 ) self-correspondence of $X$. As a selfcorrespondence it is an idempotent in the ring $\operatorname{Corr}^{0}(X)$ of degree 0 self-correspondences. If a finite group $G$ acts on $X$, any character $\chi$ of the group defines an idempotent

$$
\pi_{\chi}=\frac{1}{|G|} \sum_{g \in G} \chi(g) \Gamma_{g} \in \operatorname{Corr}^{0}(X)
$$

where $\Gamma_{g}$ is the graph of the action of $g$ on $X$. The pair $\left(X, \pi_{\chi}\right)$ is the motive canonically associated to the character $\chi$. Note that the trivial character gives the motive $\mathrm{h}(X / G)$ of the Burniat hypersurface. The main result now reads as follows:

Theorem (=Theorem 4.2) With $i: X \hookrightarrow A$ the inclusion, let $(X, G)$ be a Burniat hypersurface pair as before and let $Y=X / G$ be the corresponding generalized Burniat surface. Consider the one-dimensional space $H^{0}\left(\Omega_{A}^{3}\right)$ as a $G$-representation space, i.e. as a character $\chi_{A}$. Then

(1) For the families $\delta_{1}, \delta_{2}$ the involution $j=\iota_{1} \iota_{2} \iota_{3}$ belongs to $G$ and the motives $h(X / j)$ and $h(Y)$ are finite dimensional.

(2) For all other families, the motive $\left(X, \pi_{\chi_{A}}\right)$ is finite-dimensional. For the families $\delta_{3}, \delta_{4}$, $\delta_{11}, \delta_{12}, \delta_{16}$ this motive is just $h(Y)$.

(3) The Bloch conjecture holds for the families $\delta_{1}-\mathcal{S}_{4}$. In the remaining cases a variant of Bloch's conjecture holds, namely ${ }^{2} \operatorname{ker}\left(i_{*}: \mathrm{CH}_{0}^{\mathrm{AJ}}(X)^{\chi_{A}} \rightarrow \mathrm{CH}_{0}^{\mathrm{AJ}}(A)^{\chi_{A}}\right)=0$. For the families $\delta_{11}, \delta_{12}$ and $\delta_{16}$ this means that $\operatorname{ker}\left(i_{*}: \mathrm{CH}_{0}(X) \rightarrow \mathrm{CH}_{0}(A)\right)=0 .^{3}$

As shown in [1] the families $\delta_{11}$ and $\delta_{12}$ give two divisors in a 4-dimensional component of the Gieseker moduli space.

The above method applies also to the surfaces in this component, the so-called Sicilian surfaces so that the result for $\delta_{11}$ and $\delta_{12}$ is valid for these as well. See Remark 4.3.

1 See Sect. 2.1 for background on motives.

2 As a matter of notation, for any $G$-module $V$ we set $V \chi:=\{v \in V \mid g(v)=\chi(g) v$ for all $g \in G\}$.

3 This can also be stated directly in terms of the so-called "variable motive". See Theorem 4.2. 


\section{Preliminaries}

\subsection{Motives}

A degree $k$ (Chow) correspondences from a smooth projective variety $X$ to a smooth projective variety $Y$ is a cycle class

$$
\operatorname{Corr}^{k}(X, Y):=\mathrm{CH}^{\operatorname{dim} X+k}(X \times Y) .
$$

A correspondence of degree $k$ induces a morphism on Chow groups of the same degree and on cohomology groups (of double the degree). Correspondences can be composed and these give the morphisms in the category of Chow motives. Let me elaborate briefly on this but refer to [10] for more details.

Precisely, an effective Chow motive consists of a pair $(X, p)$ with $X$ a smooth projective variety and $p$ a degree zero correspondence which is a projector, i.e., $p^{2}=p$. Morphism between motives are induced by degree zero correspondences compatible with projectors. This procedure defines the category of effective Chow motives. Every smooth projective variety $X$ defines a motive

$$
\mathrm{h}(X)=(X, \Delta), \quad \Delta \in \mathrm{CH}^{\operatorname{dim} X}(X \times X) \text { the class of the diagonal }
$$

and a morphism $f: X \rightarrow Y$ between smooth projective varieties defines a morphism $\mathrm{h}(Y) \rightarrow \mathrm{h}(X)$ given by the transpose of the graph of $f$.

One can also use correspondences of arbitrary degrees provided one uses triples $(X, p, k)$ where $p$ is again a projector, but a morphism $f:(X, p, k) \rightarrow(Y, q, \ell)$ is a correspondence of degree $\ell-k$ compatible with projectors. Such triples define the category of Chow motives. It should be recalled that motives, like varieties have their Chow groups and cohomology groups:

$$
\begin{aligned}
\mathrm{CH}^{m}(X, p, k) & :=\operatorname{Im}\left(\mathrm{CH}^{m+k}(X) \stackrel{p_{*}}{\longrightarrow} \mathrm{CH}^{m+k}(X)\right), \\
H^{m}(X, p, k) & :=\operatorname{Im}\left(H^{m+2 k}(X) \stackrel{p_{*}}{\longrightarrow} H^{m+2 k}(X)\right) .
\end{aligned}
$$

Kimura [8] has introduced the concept finite-dimensionality for motives. If the motive of a surface $S$ is finite-dimensional, then the Bloch conjecture holds for any submotive $M$ of $S$ with $h^{2,0}(M)=0$. This is the motivation for considering the variable cohomology. In [11] it is shown that there is indeed a submotive of $S$ whose cohomology is the variable motive.

\subsection{A criterion for finite dimensional motives}

The general situation of [9] concerns smooth $d$-dimensional complete intersections $X$ inside a smooth projective manifold $M$ of dimension $d+r$ for which Lefschetz' conjecture $B(M)$ holds. This conjecture is known to hold for projective space and for abelian varieties and so in particular for the situation in this note.

Recall also (see e.g. [5, Ch. 3.2]) that in this situation, with $i: X \hookrightarrow M$ the inclusion, the fixed and variable cohomology is defined as follows.

$$
\begin{aligned}
& H_{\mathrm{fix}}^{d}(X)=\operatorname{Im}\left(i^{*}: H^{d}(M) \rightarrow H^{d}(X)\right), \\
& H_{\mathrm{var}}^{d}(X)=\operatorname{ker}\left(i_{*}: H^{d}(X) \rightarrow H^{d+2 r}(M)\right),
\end{aligned}
$$


and that one has a direct sum decomposition

$$
H^{d}(X)=H_{\text {fix }}^{d}(X) \oplus H_{\mathrm{var}}^{d}(X),
$$

which is orthogonal with respect to the intersection product. In [11] I explained that validity of $B(M)$ implies the existence of a motive $\left(X, \pi^{\mathrm{var}}\right)$ such that $\pi^{\mathrm{var}}$ induces projection onto variable cohomology.

The main input is the special case of [9, Thm. 6.5 and Cor. 6.6] for surfaces inside a threefold. It reads as follows.

Theorem 2.1 Let $M$ be a smooth projective threefold on which a finite abelian group $G$ acts. Let $\mathscr{L}$ be line bundle with $G$-action, $X \subset M a G$-invariant section and $\chi$ a character of $G$. Suppose that

(1) the conjecture $B(M)$ holds;

(2) the sections of $H^{0}(M, \mathscr{L})^{G}$ separates orbits;

(3) all characters of $G$ appear in $\operatorname{End}\left(H_{\mathrm{var}}^{2}(X)\right)$;

(4) the Chow motive $\left(M, \pi_{\chi}\right)$ is finite-dimensional;

(5) $0 \neq H_{\mathrm{var}}^{2}(X)$ and $H_{\mathrm{var}}^{2}(X)^{\chi}$ is pure of Hodge type $(1,1)$.

Then $\mathrm{CH}_{0}^{\mathrm{var}}(X)^{\chi}=0$, and the motive $\left(X, \pi_{\chi}\right)$ is finite-dimensional.

\subsection{Elliptic curves}

Let me recall the relevant facts about theta functions on an elliptic curve $E$ with period lattice $\Lambda$ generated by 1 and $\tau \in \mathfrak{h}$. Points in the elliptic curve referred to by the standard coordinate $z \in \mathbf{C}$ and the corresponding line bundle by $\mathscr{L}_{z}$. It is the bundle with $H^{0}\left(E, \mathscr{L}_{z}\right)=\mathbf{C} \vartheta_{z}$, $\vartheta_{z}$ a theta-function with simple zeros in the points $z+\Lambda$ only. Let $t_{u}: z \mapsto z+u$ be a translation of $E$. Then $\mathscr{L}_{z} \simeq t_{z}^{*} \mathscr{L}_{0}$. If one takes for $z$ one of the four two-torsion points $\epsilon \in\left\{0, \frac{1}{2}, \frac{1}{2} \tau, \frac{1}{2}+\frac{1}{2} \tau\right\}$ of $E$, the corresponding line bundles $\mathscr{L}_{\epsilon}$ have the four classical theta functions $\vartheta_{1}, \vartheta_{2}, \vartheta_{3}, \vartheta_{4}$ respectively as sections. See e.g. [6, Appendix A, Table 16] for the definitions.

Set

$$
M_{E}:=H^{0}\left(E, \mathscr{L}_{0}^{2}\right)
$$

Lemma 2.2 (i) The bundle $\mathscr{L}_{0}^{2}$ is a symmetric line bundle and all its sections are symmetric.

(ii) The translations $t_{\epsilon}$ define a faithful action of $(\mathbf{Z} / 2 \mathbf{Z})^{2}$ on $\mathscr{L}_{0}^{2}$.

(iii) The character decomposition of $M_{E}$ for this action is $(+-) \oplus(-+)$.

Proof (i) is clear.

(ii) Since $\mathscr{L}_{\epsilon}^{2} \simeq \mathscr{L}_{0}^{2}$ for all two-torsion points $\epsilon$, the functions $\vartheta_{j}^{2}$ define sections of the same bundle $\mathscr{L}_{0}^{2}$. The sections $\vartheta_{j}^{2}, j=1,2,3,4$ are characterized by having a double zero at exactly one of the four 2-torsion points. This shows in particular that the action of the group $\left\{t_{\epsilon}, \epsilon\right.$ a 2 -torsion point $\}$ is faithful on $M_{E}$.

(iii) It follows that there is a basis of two sections of $\mathscr{L}_{0}^{2}$ consisting of simultaneous eigenvectors for this action. Since the action is faithful, the character decomposition must be $(+-),(-+)$. 


\section{Surfaces inside abelian threefolds invariant under involutions}

\subsection{Products of three elliptic curves}

Consider the abelian threefold

$$
A:=E_{1} \times E_{2} \times E_{3}, \quad E_{\alpha}=\mathbf{C} / \Lambda_{\alpha}, \text { with } \quad \Lambda_{\alpha}=\mathbf{Z} \oplus \mathbf{Z} \tau_{\alpha}, \alpha=1,2,3 .
$$

Using for a fixed elliptic curve $E=\mathbf{C Z} \oplus \tau \mathbf{Z}$ the involutions

$$
\iota_{E}: z \mapsto-z, \quad t_{E}: z \mapsto-z+\frac{1}{2}, \quad \tau_{E}: z \mapsto-z+\frac{1}{2} \tau,
$$

we obtain three involutions on $A$

$$
\begin{aligned}
\iota_{\alpha} & =\iota_{E_{\alpha}} \\
\iota_{\alpha \beta} & =t_{E_{\alpha}} t_{E_{\beta}}, \\
\iota_{123} & =\tau_{E_{1}} \tau_{E_{2}} \tau_{E_{3}} .
\end{aligned}
$$

and we consider the group $(\mathbf{Z} / 2 \mathbf{Z})^{6}$ operating on $A$ as

$$
G_{0}:=\left\langle\iota_{1}, \iota_{2}, \iota_{3}, \iota_{12}, \iota_{13}, \iota_{123}\right\rangle .
$$

Lemma 3.1 The action of $G_{0}$ on holomorphic 1-forms of $A$ is given by

\begin{tabular}{llllllll}
\hline Form & $\iota_{1}$ & $\iota_{2}$ & $\iota_{3}$ & $\iota_{12}$ & $\iota_{13}$ & $\iota_{23}$ & $\iota_{123}$ \\
\hline$d z_{1}$ & - & + & + & - & - & + & - \\
$d z_{2}$ & + & - & + & - & + & - & - \\
$d z_{3}$ & + & + & - & + & - & - & - \\
\hline
\end{tabular}

Consider now the symmetric line bundle $\mathscr{L}_{A}^{2}$ where

$$
\mathscr{L}_{A}:=\sigma_{E_{1}}\left(\mathscr{L}_{0}\right) \otimes \sigma_{E_{2}}\left(\mathscr{L}_{0}\right) \otimes \sigma_{E_{3}}\left(\mathscr{L}_{0}\right),
$$

and set

$$
H^{0}\left(\mathscr{L}_{A}^{2}\right)=M_{E_{1}} \otimes M_{E_{3}} \otimes M_{E_{3}} .
$$

By Lemma 2.2 this is a representation space for $G_{0}$ which admits a basis of simultaneous eigenvectors. If $\left\{\theta_{E_{j}}^{1}, \theta_{E_{j}}^{2}\right\}$, denotes the basis of Lemma 2.2, for $M_{E_{j}}, j=1,2,3$, their products give 8 basis vectors as follows.

$$
\theta_{j_{1} j_{2} j_{3}}=\theta_{E_{1}}^{j_{1}} \cdot \theta_{E_{2}}^{j_{2}} \cdot \theta_{E_{3}}^{j_{3}}, \quad j_{k} \in 1,2 .
$$

The next result is a consequence.

Lemma 3.2 The space $H^{0}\left(\mathscr{L}_{A}^{2}\right)$ is the $G_{0}$-representation space which on the basis $\left\{\theta_{j_{1} j_{2} j_{3}}\right\}$, $j_{1}, j_{2}, j_{3} \in\{1,2\}$, is given as follows 


\begin{tabular}{|c|c|c|c|c|c|c|c|}
\hline Element & $\iota_{1}$ & $\iota_{2}$ & 13 & $\iota_{12}$ & $\iota_{13}$ & $\iota_{23}$ & $\iota_{123}$ \\
\hline$\theta_{111}$ & + & + & + & + & + & + & - \\
\hline$\theta_{211}$ & + & + & + & - & - & + & + \\
\hline$\theta_{121}$ & + & + & + & - & + & - & + \\
\hline$\theta_{112}$ & + & + & + & + & - & - & + \\
\hline$\theta_{122}$ & + & + & + & - & - & + & - \\
\hline$\theta_{212}$ & + & + & + & - & + & - & - \\
\hline$\theta_{221}$ & + & + & + & + & - & - & - \\
\hline$\theta_{222}$ & + & + & + & + & + & + & + \\
\hline
\end{tabular}

\subsection{Hypersurfaces of abelian threefolds and involutions}

Let $A$ be an abelian variety of dimension three and $\mathscr{L}$ a principal polarization so that $\mathscr{L}^{3}=$ $3 !=6$ and let $i: X \hookrightarrow A$ be a smooth surface given by a section of of $\mathscr{L}^{\otimes 2}$. The Lefschetz's hyperplane theorem gives:

$$
\begin{gathered}
i^{*}: H^{1}(A) \stackrel{\simeq}{\longrightarrow} H^{1}(X) \\
i^{*}: H^{2}(A) \stackrel{\simeq}{\longrightarrow} H_{\text {fix }}^{2}(X) \subset H^{2}(X) .
\end{gathered}
$$

Lemma 3.3 Suppose that $\iota: A \rightarrow A$ is an involution which acts on $H^{0}\left(\Omega_{A}^{1}\right)$ with p eigenvalues 1 and $n=3-p$ eigenvalues -1 . Suppose also that ı preserves $X$ and acts without fixed points on $X$. Then we have

$$
\operatorname{Tr}(\iota) \mid H_{\mathrm{var}}^{2}(X)=-29+8 p(4-p)=\left\{\begin{array}{lll}
-29 & \text { for } & p=0 \\
-5 & \text { for } & p=1 \\
3 & \text { for } & p=2 \\
-5 & \text { for } & p=3 .
\end{array}\right.
$$

Proof The assumption implies that

$$
\operatorname{Tr}(\iota) \mid H^{1}(A)=4 p-6=\left\{\begin{array}{lll}
-6 & \text { for } & p=0 \\
-2 & \text { for } & p=1 \\
2 & \text { for } & p=2 \\
6 & \text { for } & p=3
\end{array}\right.
$$

and

$$
\operatorname{Tr}(\iota) \mid H^{2}(A)=8 p(p-3)+15=\left\{\begin{array}{lll}
15 & \text { for } & p=0 \\
-1 & \text { for } & p=1 \\
-1 & \text { for } & p=2 \\
15 & \text { for } & p=3
\end{array} .\right.
$$

If $\iota$ preserves $X$ and acts without fixed points on $X$, Lefschetz' fixed point theorem gives $0=2-2 \operatorname{Tr}(\iota)\left|H^{1}(X)+\operatorname{Tr}(\iota)\right| H^{2}(X)=2-2 \operatorname{Tr}(\iota)\left|H^{1}(A)+\operatorname{Tr}(\iota)\right| H^{2}(A)+\operatorname{Tr}(\iota) \mid H_{\mathrm{var}}^{2}(X)$, and so the above calculation immediately gives the desired result.

In order to calculate the invariants on $X$, let me first consider the holomorphic two-forms in detail. 
Lemma 3.4 1. One has

$$
h_{\mathrm{var}}^{0,2}(X)=7, \quad h_{\mathrm{fix}}^{0,2}(X)=3 .
$$

2. If $X=\left\{\theta_{0}=0\right\}$, the variable holomorphic 2-forms are the Poincaré-residues along $X$ of the meromorphic 3-forms on A given by expressions of the form

$$
\frac{\theta}{\theta_{0}} d z_{1} \wedge d z_{2} \wedge d z_{3}
$$

with $\theta$ a theta-function on A corresponding to a section of $\mathscr{L}^{\otimes 2}$, and where $z_{1}, z_{2}, z_{3}$ are holomorphic coordinates on $\mathbf{C}^{3}$.

3. Suppose $\iota$ acts with the character $\epsilon \in\{ \pm 1\}$ on holomorphic three forms. Let $(p, n)$ be the dimensions of the invariant, resp. anti-invariant sections of $\mathscr{L}^{\otimes 2}$. Then $\operatorname{dim} H_{\mathrm{var}}^{2,0}(X)=$ $p-1$ if $\epsilon=1$ and $=p$ otherwise.

Proof Consider the Poincaré residue sequence

$$
0 \rightarrow \Omega_{A}^{3} \rightarrow \Omega_{A}^{3}(X) \stackrel{\text { res }}{\longrightarrow} \Omega_{X}^{2} \rightarrow 0 .
$$

In cohomology this gives

$$
0 \rightarrow H^{0}\left(\Omega_{A}^{3}\right) \rightarrow H^{0}\left(\Omega_{A}^{3}(X)\right) \stackrel{\text { res }}{\longrightarrow} H^{0}\left(\Omega_{X}^{2}\right) \rightarrow H^{1}\left(\Omega_{A}^{3}\right) \rightarrow 0 .
$$

One sees that image of the residue map coincides with $\operatorname{ker}\left(H^{0}\left(\Omega_{X}^{2}\right) \rightarrow H^{1}\left(\Omega_{A}^{3}\right)\right)$, which is the $(2,0)$-part of the variable cohomology, by definition equal to $\operatorname{ker}\left(H^{2}(X) \stackrel{i_{*}}{\longrightarrow} H^{4}(A)\right)$. Since $H^{0}\left(\Omega_{A}^{3}(X)\right)=H^{0}\left(\mathscr{L}^{\otimes 2}\right)$ the assertion 1. follows.

2. This is clear.

3. This follows directly from (4).

Corollary 3.5 The invariants of $X$ are as follows.

\begin{tabular}{lll}
\hline$b_{1}$ & $b_{2}^{\text {var }}=\left(h_{\text {var }}^{2,0}, h_{\text {var }}^{1,1}, h_{\text {var }}^{0,2}\right)$ & $b_{2}^{\text {fix }}=\left(h_{\text {fix }}^{2,0}, h_{\text {fix }}^{1,1}, h_{\text {fix }}^{0,2}\right)$ \\
\hline 6 & $43=(7,29,7)$ & $15=(3,9,3)$ \\
\hline
\end{tabular}

Proof Equation (2) gives $b_{1}(X)=b_{1}(A)=6$. To calculate $b_{2}(X)$ we observe that $c_{1}(X)=$ $-\left.2 \mathscr{L}\right|_{X}$ and $c_{2}(X)=\left.4 \mathscr{L}^{2}\right|_{X}$ so that

$$
c_{1}^{2}(X)=c_{2}(X)=\left.4 \mathscr{L}^{2}\right|_{X}=8 \mathscr{L}^{3}=48 .
$$

Since $c_{2}(X)=e(X)=2-2 b_{1}(X)+b_{2}(X)=48$, it follows that $b_{2}(X)=58$. By (3) one has $b_{2}^{\text {fix }}(X)=b_{2}(A)=15$ and so $b_{2}^{\text {var }}(X)=43$. Since $h_{\text {var }}^{2,0}=7$, the invariants for $X$ follow.

\subsection{Burniat hypersurfaces}

A Burniat hypersurface of $A=E_{1} \times E_{2} \times E_{3}$ is a surface which is invariant under a subgroup $G \subset G_{0}$ generated by 3 commuting involutions and which acts freely on $X$. Each of the involutions is a product of the involutions (1). The quotient $Y=X / G$ is called a generalized Burniat surface. In [1] one finds a list of 16 types of such surfaces, denoted $\delta_{1}, \ldots, \delta_{16}$. 
Table 1 Burniat hypersurfaces

\begin{tabular}{llllll}
\hline Type & Involution 1 & Involution 2 & Involution 3 & $G$-invariant 1 -forms & $\chi_{A}$ \\
\hline$\delta_{1}$ & $\iota_{1} \iota_{2} \iota_{3}$ & $\iota_{2} \iota_{3} \iota_{123}$ & $\iota_{3} \iota_{23}$ & None & --- \\
$\delta_{2}$ & $\iota_{1} \iota_{3} \iota_{23}$ & $\iota_{3} \iota_{13}$ & $\iota_{2} \iota_{23}$ & None & +-- \\
$\delta_{3}$ & $\iota_{1} \iota_{3} \iota_{23}$ & $\iota_{3} \iota_{123}$ & $\iota_{2} \iota_{3} \iota_{12}$ & None & +++ \\
$\delta_{4}$ & $\iota_{1} \iota_{3} \iota_{12}$ & $\iota_{3} \iota_{123}$ & $\iota_{2} \iota_{3} \iota_{23}$ & None & +++ \\
$\delta_{5}$ & $\iota_{1} \iota_{3} \iota_{13}$ & $\iota_{3} \iota_{123}$ & $\iota_{3} \iota_{23}$ & $d z_{3}$ & ++- \\
$\delta_{6}$ & $\iota_{2} \iota_{3} \iota_{123}$ & $\iota_{2} \iota_{3} \iota_{13}$ & $\iota_{3} \iota_{23}$ & $d z_{3}$ & ++- \\
$\delta_{7}$ & $\iota_{1} \iota_{3} \iota_{23}$ & $\iota_{3} \iota_{123}$ & $\iota_{2} \iota_{12}$ & $d z_{3}$ & ++- \\
$\delta_{8}$ & $\iota_{1} \iota_{3} \iota_{23}$ & $\iota_{2} \iota_{3} \iota_{123}$ & $\iota_{2} \iota_{3} \iota_{13}$ & $d z_{3}$ & +-+ \\
$\delta_{9}$ & $\iota_{1} \iota_{2} \iota_{3} \iota_{23}$ & $\iota_{3} \iota_{123}$ & $\iota_{2} \iota_{12}$ & $d z_{3}$ & +- \\
$\delta_{10}$ & $\iota_{1} \iota_{2} \iota_{3} \iota_{13}$ & $\iota_{2} \iota_{3} \iota_{123}$ & $\iota_{3} \iota_{23}$ & $d z_{3}$ & +++ \\
$\delta_{11}$ & $\iota_{1} \iota_{2} \iota_{23}$ & $\iota_{2} \iota_{123}$ & $\iota_{2} \iota_{3} \iota_{12}$ & $d z_{2}$ & +++ \\
$\delta_{12}$ & $\iota_{1} \iota_{3} \iota_{13}$ & $\iota_{3} \iota_{123}$ & $\iota_{2} \iota_{3} \iota_{23}$ & $d z_{3}$ & --- \\
$\delta_{13}$ & $\iota_{1} \iota_{2} \iota_{3} \iota_{23}$ & $\iota_{2} \iota_{3} \iota_{123}$ & $\iota_{2} \iota_{12}$ & $d z_{2}, d z_{3}$ & --- \\
$\delta_{14}$ & $\iota_{1} \iota_{13}$ & $\iota_{12} \iota_{123}$ & $\iota_{2} \iota_{23}$ & $d z_{1}, d z_{2}$ & +-+ \\
$\delta_{15}$ & $\iota_{1} \iota_{3} \iota_{13}$ & $\iota_{12} \iota_{123}$ & $\iota_{2} \iota_{3} \iota_{23}$ & $d z_{1}, d z_{2}$ & +++ \\
$\delta_{16}$ & $\iota_{1} \iota_{3} \iota_{13}$ & $\iota_{3} \iota_{12} \iota_{123}$ & $\iota_{2} \iota_{3} \iota_{23}$ & $\mathrm{All}$ & + \\
\hline
\end{tabular}

All of the surfaces are of general type with $c_{1}^{2}=6, p_{g}=q$ and $q=0,1,2,3$ and hence $e=6=2-4 q+b_{2}$ so that $b_{2}=\left(p_{g}, h^{11}, p_{g}\right)=(q, 4+2 q, q)$. There are 4 families with $q=0$ and one of these, $\mathcal{S}_{2}$ gives the classical Burniat surfaces from [4]. See Table 1.

The last column of this table gives the action of the three generators $\left(g_{1}, g_{2}, g_{3}\right)$ on $H^{0}\left(\Omega_{A}^{3}\right)$. It is calculated using Lemma 3.1. If an involution acts as the identity, there appears a " + " in the corresponding entry and else a "--"; e.g. $(+,-,-)$ means that $g_{1}=$ id but $g_{2}=g_{3}=-$ id.

In Table 2 the character spaces for the action on the forms coming from $A$ is given. It is calculated from the description of the generating involutions as given in Table 1 and the known action of 1-forms as given in Lemma 3.1. In Table 2 we use the shorthand 1 for $(+++)$; the last two columns give the Hodge numbers $\left(h^{2,0}, h^{1,1}, h^{0,2}\right)$. From the first column of this table one finds the trace of the action of these generators on $H^{0}\left(\Omega_{A}^{1}\right)$, or, alternatively, the dimensions of the eigenspaces for the eigenvalues +1 and -1 . Writing the dimensions of the $(+)$-eigenspaces as a vector according to the group elements written in the order $\left(1, g_{1}, g_{2}, g_{3}, g_{1} g_{2}, g_{1} g_{3}, g_{2} g_{3}, g_{1} g_{2} g_{3}\right)$ yields the type $\left(3, t_{1}, t_{2}, t_{3}, \ldots\right) \in \mathbf{Z}^{8}$ of the group action. This gives the first row in Table 3 below. Using Lemma 3.3, this table enables to find the multiplicity of $\chi_{A}$ in $H_{\mathrm{var}}^{2}(X){ }^{4}$

Lemma 3.6 For each of the families $\delta_{3}-\delta_{16}$ the character of $\chi_{A}$ appears with non-zero multiplicity in the variable cohomology. 5

Proof For each of the families $\delta_{3}, \delta_{4}, \delta_{11}, \delta_{12}$ and $\delta_{16}$ one has $H^{0}\left(A, \Omega_{A}^{3}\right)=(+++)$ and $\operatorname{dim} H_{\text {var }}^{1,1}(Y)=\operatorname{dim} H_{\text {var, }+++}^{1,1}(X)=1$, as one sees from Table 2 .

For the other families we argue as follows. In each case, $g \in G, g \neq 1$ act freely on $X$ and so one can apply Lemma 3.3 to find $\operatorname{Tr} g \mid H_{\mathrm{var}}^{2}(X)$, given the dimension $p(g)$ of

\footnotetext{
${ }^{4}$ We recall that $\chi_{A}$ is the isomorphism classe of the one-dimensional representation space $H^{0}\left(A, \Omega_{A}^{3}\right)$.

5 This is also true for the two remaining families, but this will not be used.
} 
Table 2 Action on forms and invariants of the generalized Burniat surfaces

\begin{tabular}{llllll}
\hline Type & $U=H^{0}\left(A, \Omega_{A}^{1}\right)$ & $H^{0}\left(A, \Omega_{A}^{2}\right)=\wedge^{2} U=W$ & $H^{1}\left(A, \Omega_{A}^{1}\right)$ & $b_{2}^{\mathrm{fix}}(Y)$ & $b_{2}^{\mathrm{var}}(Y)$ \\
\hline$\delta_{1}$ & $(-++)(--+)(-+-)$ & $(+-+)(++-)(+--)$ & $3 \cdot \mathbf{1}+2 W$ & $(0,3,0)$ & $(0,1,0)$ \\
$\delta_{2}$ & $(-++)(--+)(++-)$ & $(+-+)(-+-)(---)$ & $3 \cdot \mathbf{1}+2 W$ & $(0,3,0)$ & $(0,1,0)$ \\
$\delta_{3}$ & $(---)(--+)(++-)$ & $(++-)(--+)(---)$ & $3 \cdot \mathbf{1}+2 W$ & $(0,3,0)$ & $(0,1,0)$ \\
$\delta_{4}$ & $(+-+)(-++)(--+)$ & $(--+)(-++)(+-+)$ & $3 \cdot \mathbf{1}+2 W$ & $(0,3,0)$ & $(0,1,0)$ \\
$\delta_{5}$ & $(+-+)(+--)+\mathbf{1}$ & $(+-+)(+--)(++-)$ & $3 \cdot \mathbf{1}+2 W$ & $(0,3,0)$ & $(1,3,1)$ \\
$\delta_{6}$ & $(--+)(+--)+\mathbf{1}$ & $(--+)(+--)(-+-)$ & $3 \cdot \mathbf{1}+2 W$ & $(0,3,0)$ & $(1,3,1)$ \\
$\delta_{7}$ & $(---)(--+)+\mathbf{1}$ & $(---)(--+)(++-)$ & $3 \cdot \mathbf{1}+2 W$ & $(0,3,0)$ & $(1,3,1)$ \\
$\delta_{8}$ & $(---)(-+-)+\mathbf{1}$ & $(---)(-+-)(+-+)$ & $3 \cdot \mathbf{1}+2 W$ & $(0,3,0)$ & $(1,3,1)$ \\
$\delta_{9}$ & $(---)(+-+)+\mathbf{1}$ & $(---)(+-+)(-+-)$ & $3 \cdot \mathbf{1}+2 W$ & $(0,3,0)$ & $(1,3,1)$ \\
$\delta_{10}$ & $(+-+)(-+-)+\mathbf{1}$ & $(+-+)(-+-)(---)$ & $3 \cdot \mathbf{1}+2 W$ & $(0,3,0)$ & $(1,3,1)$ \\
$\delta_{11}$ & $2 \cdot(---)+\mathbf{1}$ & $2 \cdot(---)+\mathbf{1}$ & $3 \cdot \mathbf{1}+2 W$ & $(1,5,1)$ & $(0,1,0)$ \\
$\delta_{12}$ & $2 \cdot(+-+)+\mathbf{1}$ & $2 \cdot(+-+)+$ & $3 \cdot \mathbf{1}+2 W$ & $(1,5,1)$ & $(0,1,0)$ \\
$\delta_{13}$ & $(---)+2 \cdot \mathbf{1}$ & $2 \cdot(---)+\mathbf{1}$ & $3 \cdot \mathbf{1}+2 W$ & $(1,5,1)$ & $(1,3,1)$ \\
$\delta_{14}$ & $(---)+2 \cdot \mathbf{1}$ & $2 \cdot(---)+\mathbf{1}$ & $3 \cdot \mathbf{1}+2 W$ & $(1,5,1)$ & $(1,3,1)$ \\
$\delta_{15}$ & $(+-+)+2 \cdot \mathbf{1}$ & $2 \cdot(+-+)+\mathbf{1}$ & $3 \cdot \mathbf{1}+2 W$ & $(1,5,1)$ & $(1,3,1)$ \\
$\delta_{16}$ & $3 \cdot \mathbf{1}$ & $3 \cdot \mathbf{1}$ & $3 \cdot \mathbf{1}+2 W$ & $(3,9,3)$ & $(0,1,0)$ \\
\hline
\end{tabular}

Table 3 Trace vectors

\begin{tabular}{lllll}
\hline Type & Type $H^{0}\left(A, \Omega_{A}^{1}\right)$ & Trace vect. $H_{\text {var }}^{2}(X)$ & Trace vect. $H^{0}\left(A, \Omega_{A}^{3}\right)$ & Mult. $\chi_{A}$ \\
\hline$\delta_{5}$ & $(3|312| 122 \mid 2)$ & $(43|-53-5|-533 \mid 3)$ & $(1|1|-1|1-1-1|-1)$ & 2 \\
$\delta_{6}$ & $(3|212| 212 \mid 3)$ & $(43|3-53| 3-53 \mid-5)$ & $(1|-11-1|-11-1 \mid 1)$ & 2 \\
$\delta_{7}$ & $(3|112| 322 \mid 2)$ & $(43|-5-53|-533 \mid 3)$ & $(1|1|-1|1-1-1|-1)$ & 2 \\
$\delta_{8}$ & $(3|121| 232 \mid 2)$ & $(43|-53-5| 3-53 \mid 3)$ & $(1|1-1| 1|-11-1|-1)$ & 2 \\
$\delta_{9}$ & $(3|212| 232 \mid 1)$ & $(43|3-53| 3-53 \mid-5)$ & $(1|-11-1|-11-1 \mid 1)$ & 2 \\
$\delta_{10}$ & $(3|222| 131 \mid 2)$ & $(43|333|-55-5 \mid 3)$ & $(1|-1-1-1| 1|1|-1)$ & 2 \\
$\delta_{13}$ & $(3|222| 333 \mid 2)$ & $(43|333|-5-5-5 \mid 3)$ & $(1|-1-1-1| 1|1|-1)$ & 2 \\
$\delta_{14}$ & $(3|222| 333 \mid 2)$ & $(43|333|-5-5-5 \mid 3)$ & $(1|-1-1-1| 11 \mid-1)$ & 2 \\
$\delta_{15}$ & $(3|323| 232 \mid 2)$ & $(43|-53-5| 3-53 \mid 3)$ & $(1|1-11|-11-1 \mid-1)$ & 2 \\
\hline
\end{tabular}

the (+1)-eigenspace of $H^{0}\left(\Omega_{A}^{1}\right)$. This type is given in Table 3. The next column gives the corresponding trace vector. Then follows the trace vector of $\chi_{A}$. Now apply the trace formula for the multiplicity of an irreducible representation inside a given representation (see e.g. $[12, \S 2.3])$ : just take the "dot" product of the two trace vectors and divide by the order of the group. Let me do this explicitly for the family $\delta_{5}$. The trace vector for $H_{\mathrm{var}}^{2}(X)$ is $(43,-5,-5,3,-5,3,3,3)$, the first number being $\operatorname{dim} H_{\mathrm{var}}^{2}(X)$. The representation $\chi_{A}=$ $(++-)$ has trace vector $(1,1,1,-1,1,-1,-1,-1)$ and the trace formula reads

$$
\frac{1}{8}(43-5-5-3-5-3-3-3)=2 .
$$




\section{The main result}

In this section I shall show that the main theorem 4.2 below follows upon application of Theorem 2.1. First an auxiliary result.

Lemma 4.1 Consider for each of the families $\delta_{1}-\delta_{16}$ the space of theta-functions $H^{0}\left(\mathscr{L}_{A}^{2}\right)$ as $G$-representation space. This 8-dimensional space is the direct sum for all 8 characters of $G$ except for the families $\delta_{1}$ and $\delta_{2}$. For these families we have

- for $\delta_{1}$ we have $H^{0}\left(\mathscr{L}_{A}^{2}\right)=2((+++)+(++-)+(+-+)+(+--))$,

- for $\delta_{2}$ we have $H^{0}\left(\mathscr{L}_{A}^{2}\right)=2((+++)+(+-+)+(-+-)+(--))$.

Proof This follows from the $G$-action on the basis $\theta_{j_{1}, j_{2} j_{3}}$ for $H^{0}\left(\mathscr{L}_{A}^{2}\right)$ which can be deduced from Lemma 3.2. I shall work this out for two cases: the family $\delta_{2}$, and for the family $\delta_{6}$. For $\delta_{2}$ we have $g_{1}=\iota_{1} \iota_{3} \iota_{23}, g_{2}=\iota_{3} \iota_{13}$ and $g_{3}=\iota_{2} \iota_{23}$ and for $\delta_{6}$ we have $g_{1}=\iota_{2} \iota_{3} \iota_{123}$, $g_{2}=\iota_{2} \iota_{3} \iota_{13}$ and $g_{3}=\iota_{3} \iota_{23}$, and the action of these involutions is given in the following table.

\begin{tabular}{lllllll}
\hline Element & $g_{1}=\iota_{1} \iota_{3} \iota_{23}$ & $g_{2}=\iota_{3} \iota_{13}$ & $g_{3}=\iota_{2} \iota_{23}$ & $g_{1}=\iota_{2} \iota_{3} \iota_{123}$ & $g_{2}=\iota_{2} \iota_{3} \iota_{13}$ & $g_{3}=\iota_{3} \iota_{2} 3$ \\
\hline$\theta_{111}$ & + & + & + & - & + & + \\
$\theta_{211}$ & + & - & + & + & - & + \\
$\theta_{121}$ & - & + & - & + & + & - \\
$\theta_{112}$ & - & - & - & + & - & - \\
$\theta_{122}$ & + & - & + & - & - & + \\
$\theta_{212}$ & - & + & - & - & + & - \\
$\theta_{221}$ & - & - & - & - & - & - \\
$\theta_{222}$ & + & + & + & + & + & + \\
\hline
\end{tabular}

Theorem 4.2 Let $(X, G)$ be a Burniat hypersurface pair as before and let $Y=X / G$ be the corresponding generalized Burniat surface. Consider the one-dimensional space $H^{0}\left(\Omega_{A}^{3}\right)$ as a $G$-representation space, i.e. as a character $\chi_{A}$. Then

(1) For the families $\delta_{1}, \delta_{2}$ the involution $j=\iota_{1} \iota_{2} \iota_{3}$ belongs to $G$ and the motives $h(X / j)$ and $h(Y)$ are finite dimensional.

(2) For all other families, the motive $\left(X, \pi_{\chi_{A}}\right)$ is finite-dimensional. For the families $\delta_{3}, \delta_{4}$, $\delta_{11}, \delta_{12}$ and $\delta_{16}$ the latter motive is just $h(Y)$.

(3) The Bloch conjecture holds for the families $\delta_{1}-S_{4}$. In the remaining cases a variant of Bloch's conjecture holds, namely $\mathrm{CH}_{0}^{\mathrm{var}}(X)^{\chi_{A}}=0$. For the families $\delta_{11}, \delta_{12}$ and $\delta_{16}$ this means that $\mathrm{CH}_{0}^{\mathrm{var}}(X)=0$.

Proof (1) For the family $\delta_{2}$ this is [9, Example 7.3]. The same proof goes through for the family $\delta_{1}$.

(2) The conditions (1), (2) and (4) of Theorem 2.1 are verified. Condition (5) is a consequence of Lemma 3.4.3. Indeed, if all characters in the 8-dimensional space $H^{0}\left(A, \mathscr{L}^{2}\right)$ appear once, this result implies that there is one character missing in $H_{\mathrm{var}}^{0,2}(X)$, namely the character $\chi_{A}$ for the holomorphic three-forms on $A$. So for this character space condition (5) holds. As to (3), Lemma 4.1 states that in this case all characters appear in $H_{\mathrm{var}}^{2}(X)$ except maybe this missing character $\chi_{A}$. But its multiplicity has been calculated in Table 3 . It is non-zero and so condition (3) holds as well.

(3) This is one of the assertions of Theorem 2.1. 
Remark 4.3 Recall the following definition from [1]: a Sicilian surface is a minimal surface $S$ of general type with numerical invariants $p_{g}(S)=q(S)=1, c_{1}^{2}(S)=6$ for which, in addition, there exists an unramified double cover $\hat{S} \rightarrow S$ with $q(\hat{S})=3$, and such that the Albanese morphism $\hat{\alpha}: \hat{S} \rightarrow \operatorname{Alb}(\hat{S})$ is birational to its image $Z$, a divisor in its Albanese variety with $Z^{3}=12$. In loc. cit. one finds the following explicit construction. Let $T=\mathbf{C}^{2} / \Lambda_{2}, \Lambda_{2}=\mathbf{Z}^{2} \oplus \tau_{1} \mathbf{Z} \oplus \mathbf{Z} \tau_{2}$ be an Abelian surface with a (1, 2)-polarization $\mathscr{L}_{2}$ and let $E=\mathbf{C} / \Lambda, \Lambda=\mathbf{Z} \oplus \tau \mathbf{Z}$ be an elliptic curve. Consider the sections of the line bundle $\mathscr{L}=\mathscr{L}_{0}^{\otimes 2} \otimes \mathscr{L}_{2}$ on $A:=E \times T$ that are invariant under the action of the bi-cyclic group $K$ generated by $(e, a) \mapsto\left(e+\frac{1}{2} \tau,-a+\frac{1}{2} \tau_{1}\right)$ and $(e, a) \mapsto\left(e+\frac{1}{2}, a+\frac{1}{2} \tau_{2}\right)$. These sections define hypersurfaces $X \subset A$ and the quotient $Y=X / K$ is a Sicilian surface and all such surfaces are obtained in this way.

Let me consider the invariants. Note that $p_{g}(Y)=q(Y)=1, c_{1}^{2}(Y)=6$ implies that $h^{1,1}(Y)=h^{1,1}(X)_{++}=6$. In the same manner as for the families $\delta_{11}$ and $\delta_{12}$ one shows that $h_{\mathrm{var},++}^{2,0}=0$ so that $H^{1,1}(X)_{\mathrm{var},++}=H^{2}(X)_{\mathrm{var},++}$. Moreover, one finds $h^{1,1}(X)_{\mathrm{var},++}=1$. In the course of the proof of [1, Theorem 6.1] it is remarked that $H^{0}(A, \mathscr{L})=(++) \oplus(+-) \oplus$ $(-+) \oplus(--)$. Clearly, $H^{0}\left(\Omega_{A}^{3}\right)$ is invariant under $K$ and the residue calculus (cf. Lemma 3.4) shows that $H_{\mathrm{var}}^{2,0}=(+-) \oplus(-+) \oplus(--)$ and so the "missing character" $\chi_{A}$ is the trivial character. Since $h^{1,1}(X)_{\mathrm{var},++}=1$ this missing character is present in $H^{2}(X)_{\mathrm{var}}$ and by Theorem 2.1 it follows that for Sicilian surfaces $Y$, one has $\mathrm{CH}_{0}^{\mathrm{var}}(Y)=0$ and the motive $\mathrm{h}(Y)$ is finite-dimensional.

Acknowledgements Thanks to Robert Laterveer for his interest and remarks. I also want to thank the referee for her helpful hints to improve the presentation; she also carefully checked the calculations, thereby saving me from embarrassment.

Open Access This article is distributed under the terms of the Creative Commons Attribution 4.0 International License (http://creativecommons.org/licenses/by/4.0/), which permits unrestricted use, distribution, and reproduction in any medium, provided you give appropriate credit to the original author(s) and the source, provide a link to the Creative Commons license, and indicate if changes were made.

\section{References}

1. Bauer, I., Catanese, F., Frapporti, D.: Generalized Burniat type surfaces and Bagnera-deFranchis varieties. J. Math. Sci. Univ. Tokyo 22(2-15), 55-111 (2015). arXiv:1409.1285v2

2. Bauer, I., Frapporti, D.: Bloch's conjecture for generalized Burniat type surfaces with $p_{g}=0$. Rend. Circ. Mat. Palermo (2) 64, 27-42 (2015)

3. Bloch, S.: Lectures on algebraic cycles, Duke University Press Durham 1980, new edition: New Mathematical Monographs 16, Cambridge Univ. Press (2010)

4. Burniat, P.: Sur les surfaces de genre $P_{12}>1$. Ann. Mat. Pura Appl. 71, 1-24 (1966)

5. Carlson, J., Müller-Stach, S., Peters, C.: Period Mappings and Period Domains. Cambridge Studies in Advanced Mathematics, 2nd edn. Cambridge University Press, Cambridge (2017)

6. Encyclopedic dictionary of mathematics. Vol. I-IV. Translated from the Japanese. Second edition. Edited by Kiyosi Ito. MIT Press, Cambridge,MA, 1987. xx+2148 pp. ISBN: 0-262-09026-000A

7. Inoue, M.: Some new surfaces of general type. Tokyo J. Math. 17, 295-319 (1994)

8. Kimura, S.: Chow groups are finite dimensional, in some sense. Math. Ann. 331, 173-201 (2005)

9. Laterveer, R., Nagel, J., Peters, C.: On complete intersections in varieties with finite-dimensional motive. arXiv:1709.10259 [math.AG]. To appear in Quarterly Journal of Mathematics

10. Murre, J., Nagel, J., Peters, C.: Lectures on the Theory of Pure Motives. University Lecture Series. American Mathematical Society, Providence (2013)

11. Peters, C.: On a motivic interpretation of primitive, variable and fixed cohomology. Math. Nachrichten. https://doi.org/10.1002/mana.201800011

12. Serre, J.-P.: Linear Representations of Finite Groups Graduate Texts in Math. Springer, New York (1977). etc 